\title{
Urinary System Findings Domain
}

National Cancer Institute

\section{Source}

National Cancer Institute. Urinary System Findings Domain. NCI Thesaurus. Code C102726.

A subject domain utilized for the submission of information encompassing and representing data, vocabulary or records related to urinary system findings. 